\title{
T-Cell Epitopes in Type 1 Diabetes Autoantigen Tyrosine Phosphatase IA-2: Potential for Mimicry with Rotavirus and Other Environmental Agents
}

\author{
Margo C. Honeyman, Natalie L. Stone, \\ and Leonard C. Harrison \\ Autoimmunity and Transplantation Division, The Walter and Eliza \\ Hall Institute of Medical Research, Parkville, Australia \\ Communicated by L. C. Harrison. Accepted February 17, 1998.
}

\begin{abstract}
The tyrosine phosphatase IA-2 is a molecular target of pancreatic islet autoimmunity in type 1 diabetes. T-cell epitope peptides in autoantigens have potential diagnostic and therapeutic applications, and they may hold clues to environmental agents with similar sequences that could trigger or exacerbate autoimmune disease. We identified 13 epitope peptides in IA- 2 by measuring peripheral blood T-cell proliferation to 68 overlapping, synthetic peptides encompassing the intracytoplasmic domain of IA-2 in six at-risk type 1 diabetes relatives selected for HLA susceptibility haplotypes.

The dominant epitope, VIVMLTPLVEDGVKQC (aa 805820), which elicited the highest $\mathrm{T}$-cell responses in all at-risk relatives, has $56 \%$ identity and $100 \%$ similarity over 9 amino acids (aa) with a sequence in VP7, a major immunogenic protein of human rotavirus. Both peptides bind to HLA-DR4 $\left({ }^{*} 0401\right)$ and are deduced to present
\end{abstract}

identical aa to the T-cell receptor. The contiguous sequence of VP7 has $75 \%$ identity and $92 \%$ similarity over 12 aa with a known $\mathrm{T}$-cell epitope in glutamic acid decarboxylase (GAD), another autoantigen in type 1 diabetes. This dominant IA-2 epitope peptide also has $75-45 \%$ identity and $88-64 \%$ similarity over $8-14$ aa to sequences in Dengue, cytomegalovirus, measles, hepatitis $\mathrm{C}$, and canine distemper viruses, and the bacterium Haemophilus influenzae. Three other IA-2 epitope peptides are $71-100 \%$ similar over $7-12$ aa to herpes, rhino-, hanta- and flaviviruses. Two others are $80-82 \%$ similar over $10-11$ aa to sequences in milk, wheat, and bean proteins. Further studies should now be carried out to directly test the hypothesis that T-cell activation by rotavirus and possibly other viruses, and dietary proteins, could trigger or exacerbate $\beta$-cell autoimmunity through molecular mimicry with IA-2 and (for rotavirus) GAD.

\section{Introduction}

The recently identified pancreatic islet autoantigen in type 1 diabetes, IA-2, is a $106 \mathrm{kD}$ member of the protein tyrosine phosphatase family $(1,2)$ and an integral membrane protein of neuroendocrine secretory granules (3). Circulating autoantibodies that recognize predominantly the cy-

Address correspondence and reprint requests to: Dr. Margo C. Honeyman or Leonard C. Harrison, The Walter and Eliza Hall Institute of Medical Research, Royal Melbourne Hospital, Victoria 3050, Australia. Phone: 61-3-9345 2555; Fax: 61-3-9347 0852; E-mail: honeyman@wehi.edu.au toplasmic domain of IA-2 can be detected in up to $88 \%$ of people with recently diagnosed type 1 diabetes and in about half of islet cell antibody (ICA)-positive, first-degree type 1 diabetes relatives in whom they indicate high risk for clinical disease (4). The cytoplasmic domain of IA-2 has $80 \%$ sequence identity with another tyrosine phosphatase, IAR (5), also known as IA-2 $\beta$ (6) or phogrin (7), which also reacts with antibodies in type 1 diabetes (8). T-cell proliferative responses to IA-2 were reported to be increased in at-risk relatives and in people with recently di- 
agnosed type 1 diabetes (9). T-cell epitope peptides in autoantigens have potential diagnostic and therapeutic applications and may hold clues to environmental agents that could trigger or exacerbate autoimmune disease. We identified T-cell epitope peptides within the intracytoplasmic domain of IA-2 and examined them for sequence similarities with microorganisms and dietary proteins as a basis for molecular mimicry.

\section{Materials and Methods}

\section{Subjects}

Peripheral blood was obtained from six at-risk, ICA-positive first-degree relatives of people with type 1 diabetes ( 4 male, 2 female, mean age $28.5 \pm 15.0$, range 10-50) and two healthy control subjects ( 2 males, ages 30 and 48 ). Subjects were selected for type 1 diabetes-associated HLA haplotypes, i.e., DR4-DQ8 homozygous (two atrisk relatives, one control), DR3-DQ2 homozygous (two at-risk relatives, one control), and DR4-DQ8/DR3-DQ2 heterozygous (two at-risk relatives). All relatives had antibodies to IA-2. Within 14 months of the study, both DR4-DQ8 homozygous relatives developed clinical type 1 diabetes and the first-phase insulin release in response to intravenous glucose in both DR3DQ2 homozygous relatives fell to below the first percentile, indicating imminent clinical disease. The study was approved by the Ethics Committee at the Institute and was conducted with informed consent.

\section{IA-2 Antibody Assay}

Antibodies to IA-2 (IA-2 Ab) were measured by immunoprecipitation of ${ }^{35} \mathrm{~S}$-methionine-labeled full-length recombinant IA-2 (4). IA-2 was synthesized from cDNA cloned into the EcoRl site of the Bluescript KS vector in the presence of ${ }^{35} \mathrm{~S}$ methionine, in the TNT-reticulocyte lysate system (Promega, Madison, WI). The assay has a sensitivity of $60 \%$ and specificity of $97 \%$ for newly diagnosed type 1 diabetes and was standardized in the Third Combined Autoantibody Workshop (Florida, 1996). The upper limit of the normal range (mean +3 SD of healthy controls) is 3 units. Results were expressed as a percentage of counts precipitated by a reference serum. The intra- and interassay coefficients of variation (CV) were $1 \%$ and $4 \%$, respectively.

\section{Tissue Typing}

HLA alleles were typed by the standard microlymphocytotoxic method for all recognized HLA class I alleles. HLA-DR and DQ types were determined by sequence-specific oligotyping, following the International Histocompatibility Workshop protocol.

\section{Peptides}

A set of 68 16-mer peptides was synthesized (Chiron Technologies, Melbourne, Australia). Sixty-two peptides overlapping by 10 aa spanned the cytoplasmic domain of human IA-2 (aa 601979). Six additional 16-mers (aa 713-728, 779794, 795-810, 831-846, 845-860, 959-974) covered sequences predicted to bind to DR4 $\left({ }^{*} 0401\right)(10,11)$. Peptides were synthesized by Fmoc chemistry and solid-phase synthesis, with free amino and free acid carboxy-termini, using base-labile or acid-labile resins as appropriate. Each peptide was dissolved in $100 \mu \mathrm{l} 40 \%$ acetonitrile in degassed phosphate buffered saline (PBS) and shaken at $4^{\circ} \mathrm{C}$ overnight, checked for solubility, sonicated in an immersion sonicator for up to $60 \mathrm{~min}$ at room temperature (RT) if necessary, then diluted to $1 \mathrm{mg} / \mathrm{ml}$ in PBS. Each peptide was dispensed into 12 wells of a sterile 96-well round-bottomed tissue culture tray (Linbro) and stored at $-80^{\circ} \mathrm{C}$.

\section{HLA-DR4 Binding}

Peptide binding to purified DR4(*0401) was measured directly by a competition enzyme linked immunosorbant assay (ELISA), as previously described $(12,13)$.

\section{T-Cell Proliferation Assays}

Peripheral blood mononuclear cells (PBMC) were separated from heparinized venous blood by Ficoll-Hypaque density centrifugation, washed twice in RPMI 1640 medium, and diluted to $10^{6}$ cells $/ \mathrm{ml}$ in RPMI1640 medium containing $10 \%$ autologous serum, $20 \mathrm{mM}$ Hepes, and $10^{-5} \mathrm{M} 2$-mercaptoethanol (complete medium). Two $\times 10^{5}$ cells were added in $200 \mu \mathrm{l}$ of complete medium to each well of freshly thawed, peptide-containing 96-well trays. Each peptide was tested at $10 \mu \mathrm{g} / \mathrm{ml}$ in replicates of 12 . The first row of each tray contained six wells without antigen (basal) and six wells with 1.8 Lyons flocculating units (Lfu) $/ \mathrm{ml}$ of preservativefree tetanus toxoid (Commonwealth Serum Lab- 
RQHARQQDKERLAALGPEGAHGDTTFEYQDLCRQHMATKSLFNRAEGPPEPSRVSSVSSQFSDAAQASPSSHSSTP

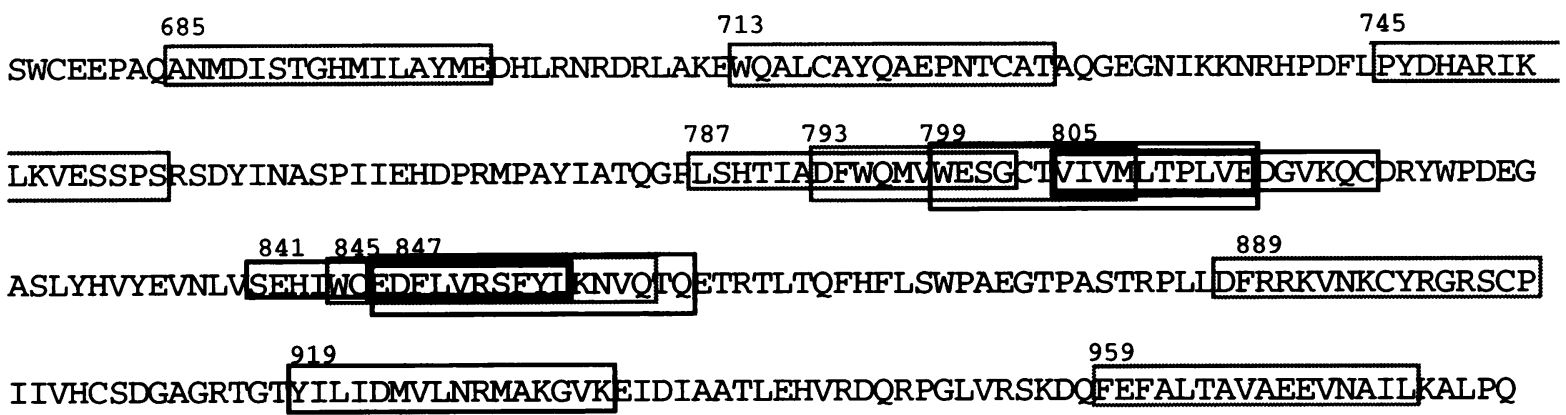

Fig. 1. Summary of identified T-cell epitope peptides in tyrosine phosphatase IA-2. Bolded boxes contain sequences common to overlapping epitope peptides; unbolded boxes contain epitope

oratories, Melbourne); the last row of each tray contained six wells with $0.18 \mathrm{Lfu}$ of tetanus toxoid and six wells without antigen. After incubation for 6 days in $5 \% \mathrm{CO}_{2}$ at $37^{\circ} \mathrm{C}, 37 \mathrm{kBq}$ ${ }^{3} \mathrm{H}$-thymidine (ICN, $2.5 \mathrm{TBq} / \mathrm{mmol}$ ) was added per well; the cells were harvested semi-automatically $7 \mathrm{hr}$ later and ${ }^{3} \mathrm{H}$-thymidine incorporation measured by liquid scintillation counting. As Tcell responses to peptides approximate a Poisson rather than a normal distribution, proliferation was expressed as the percent positive of the 12 replicate wells. Positive wells were defined as having cpm $>$ mean +2 SD of the 12 basal wells for that plate. A T-cell response to a peptide was defined as positive wells $\geq 40 \%$; this threshold was the mean +2 SD of the 136 responses of the controls to all peptides (mean 6\%, SD 17\%). $\mathrm{T}$-cell epitopes were defined as being within peptides that elicited a response in two at-risk relatives with the same HLA haplotype, e.g., both DR4-DQ8 homozygotes, or one DR4-DQ8 homozygote and at least one DR3-DQ2/DR4-DQ8 heterozygote. The reproducibility of T-cell proliferation to tetanus $(1.8 \mathrm{Lfu} / \mathrm{ml})$ was tested by repeat assays weekly for 4 weeks in three subjects; intra-assay CVs ranged from $13.1 \%$ to $18.9 \%$ and the interassay CV from $14.2 \%$ to $26.2 \%$.

\section{Database Searches}

Similarities to the sequences of epitope peptides or their common overlapping sequences were sought using FASTA 2 software. Databases searched were Genbank (GBTrans) (1997), Swissprot (1997), Protein Research Foundation peptides presented by both DR3-DQ2 and DR4-DQ8 haplotypes; stippled boxes contain epitope peptides presented only by the DR4-DQ8 haplotype.

of Japan (PRFJ) (1997), and Ooi Japan (OOIJ) (1983). No statistical significance was assigned to search results because the databases included many sequences homologous to IA-2, e.g., B220, CD45, IA-2 $\beta$, phogrin, IAR, and other tyrosine phosphatases. Infectious or dietary agents were selected on the basis of potential biological relevance, as in other studies (14), from the first 60 best matches in the PRFJ and OOIJ databases and from the first 100 in the larger GBTrans and Swissprot databases.

\section{Results}

From 68 16-mer peptides encompassing cytoplasmic IA-2, 11 peptides (from aa 685, 713, 745, $787,793,805,841,845,847,919$, and 959) elicited T-cell responses in relatives homozygous for DR4-DQ8 and two peptides (from aa 799 and 889) elicited responses in one DR4-DQ8 homozygous and one DR4-DQ8/DR3-DQ2 heterozygous relative (Fig. 1). All these epitope peptides bound to HLA-DR4 (Table 1). Five peptides (from aa 799, 805, 841, 847, 919) elicited responses in the DR3-DQ2 homozygous relatives, and the first four of these also elicited responses in the matched control. Notably, peptide EDF LVRSFYLKNVQTQ (aa 847-862) elicited responses in both DR3-DQ2 and DR4-DQ8 homozygous controls, as well as in one DR3-DQ2 homozygous, one heterozygous, and both DR4DQ8 homozygous at-risk relatives. Peptide VIVMLTPLVEDGVKQC (aa 805-820) elicited a response in all at-risk relatives and in the DR3DQ2 homozygous control; in each case it was the 


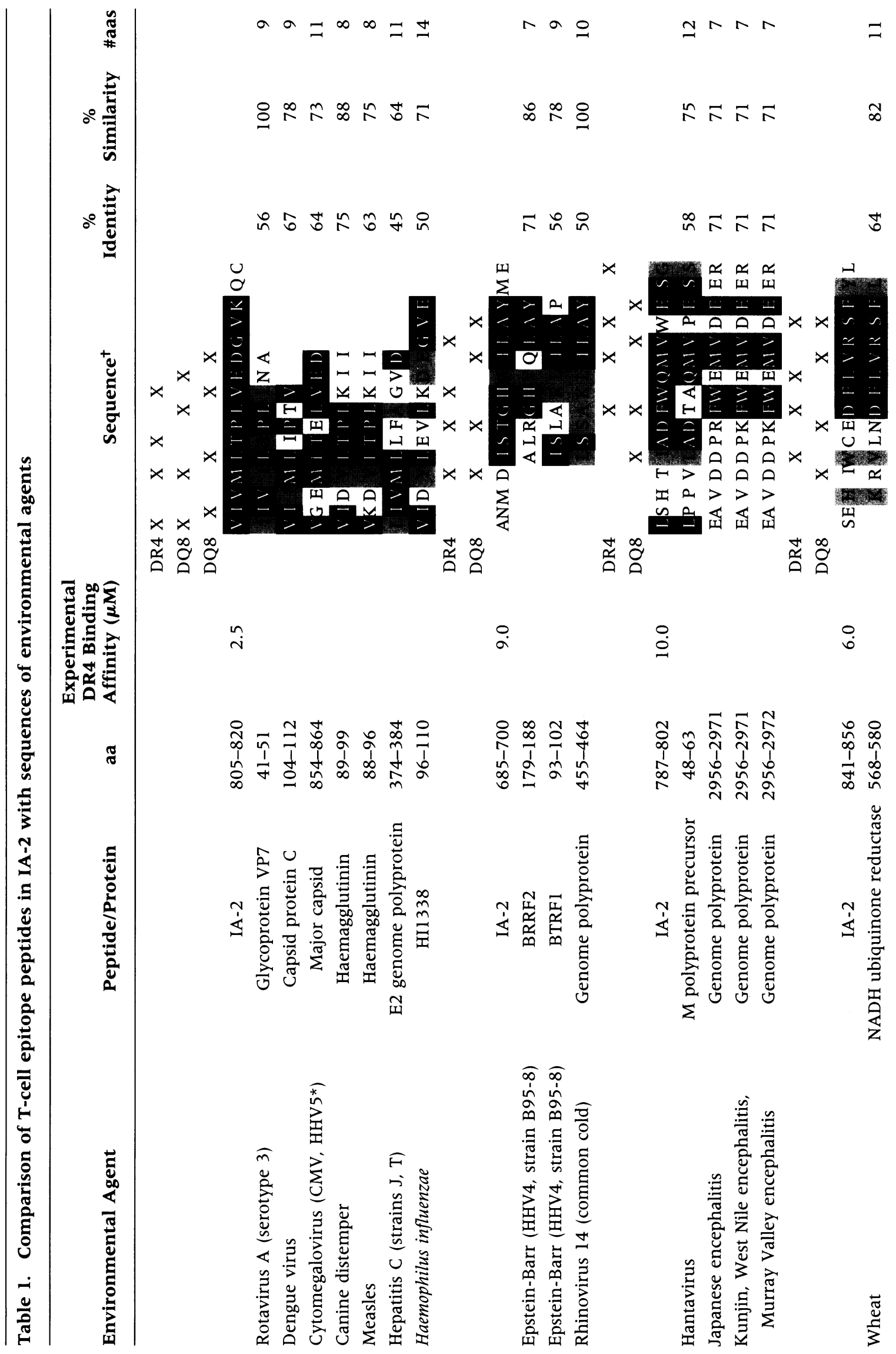




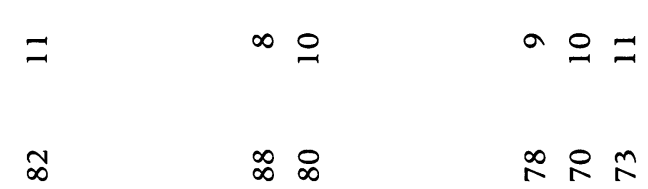

Iำ

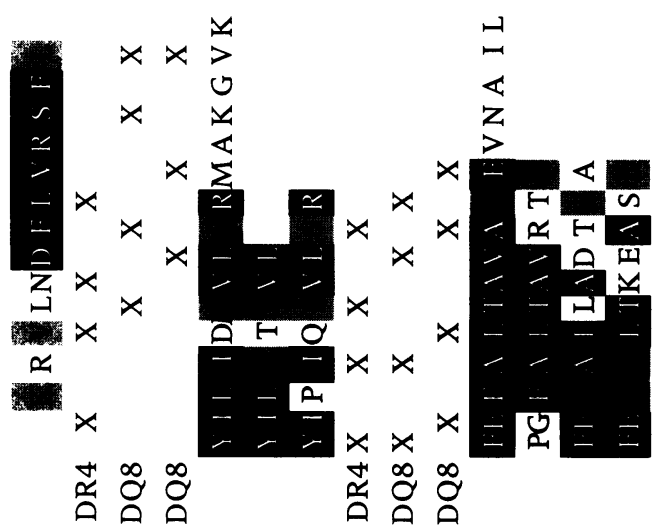

$\stackrel{n}{2} \stackrel{0}{-1}$

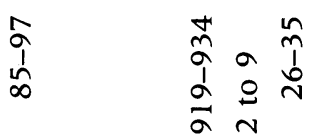

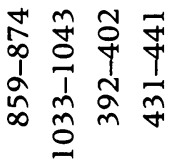

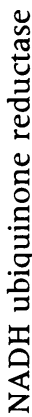
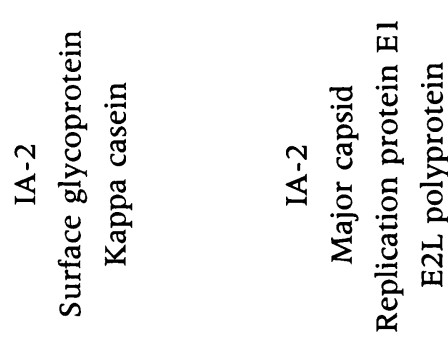

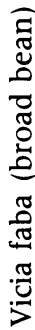

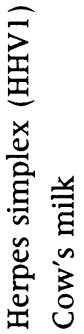

highest response (relatives, $86 \pm 20 \%$ positive wells; control $100 \%$ positive wells). Alignment of the IA-2 epitope peptides with related sequences in human tyrosine kinase IAR (5) revealed identities of $86 \pm 12 \%$ and similarities of $92 \pm 10 \%$ (mean \pm SD).

Epitope peptides of IA-2 shared identity or similarity with several environmental agents (Table 1). The dominant epitope peptide VIVML TPLVEDGVKQC had sequence identities of 75$45 \%$ and similarities of $100-64 \%$ over $8-11$ aa to sequences within the VP7 protein of rotavirus (serotype 3, strain $\mathrm{P}$ ), the capsid protein $\mathrm{C}$ of Dengue flavivirus, the major capsid protein of human cytomegalovirus, the hemagglutinin proteins of canine distemper virus (known to infect humans) and the closely related measles virus, and the $\mathrm{E} 2$ protein of hepatitis $\mathrm{C}$ virus. It also had $50 \%$ identity and $71 \%$ similarity over 14 aa with the HI 1338 protein of the bacterium Haemophilus influenzae. Most of the sequence similarities were in the region of overlap VIVMLTPLVE (aa 805-814) with the preceding epitope peptide (aa 799-814). The rotavirus VP7 protein also had $75 \%$ identity and $92 \%$ similarity over 12 aa (aa $18-29$ ) (or $75 \%$ and $100 \%$ over 9 aa) to GAD65 (aa 117-128), and GAD67 (aa 123-134) (Table 2).

Peptide aa $685-700$ had $56-71 \%$ identity and $78-86 \%$ similarity to the BTRF1 and BRRF2 proteins of Epstein-Barr virus, and $50 \%$ identity and $100 \%$ similarity over 10 aa to the genome polyprotein of rhinovirus 14 , the common cold virus.

Peptide aa $787-802$ had $58 \%$ identity and $75 \%$ similarity over 12 aa to the $M$ polyprotein precursor of hantavirus, and $71 \%$ identity and similarity over 7 aa to sequences within the genome polyprotein of other members of the Flavivirus family, i.e., Japanese encephalitis, Kunjin, West Nile, and Murray Valley encephalitis viruses. Most of the sequence similarities were in the region of overlap DFWQMVWESG (aa 793802 ) with the succeeding epitope peptide (aa 793-808).

Peptide aa $841-856$ had $64 \%$ identity and $82 \%$ similarity over 11 aa to proteins in wheat and broad beans, and epitope peptide aa 919934 had $60 \%$ identity and $80 \%$ similarity over 10 aa to kappa casein in cow's milk. Most of the sequence similarities were in the region of overlap EDFLVRSFYL (aa 847-856) with the two succeeding epitope peptides (aa 845-860, 847856).

Peptide aa 919-934 had 63\% identity and 


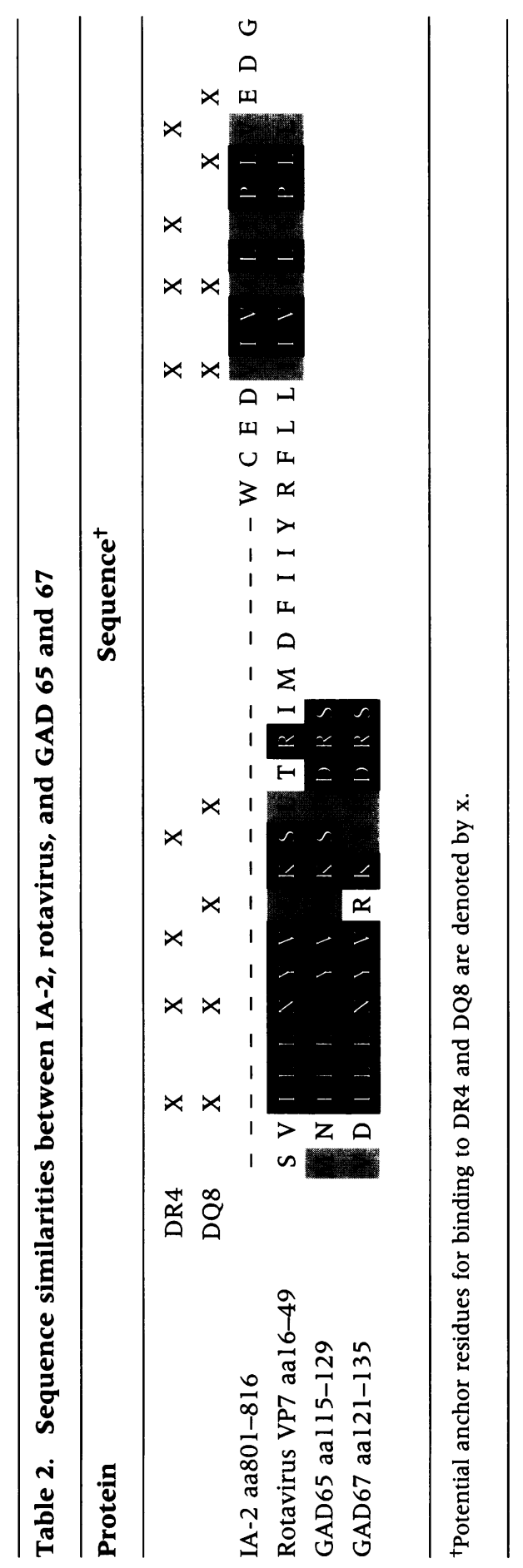

$88 \%$ similarity over 8 aa to the surface glycoprotein of herpes simplex virus. Peptide aa 959974 had $67 \%$ identity and $78 \%$ similarity over 9 aa to the major capsid protein of cytomegalovirus (HHV5) and herpes saimiri virus (which can infect human lymphocytes), and $50 \%$ identity and $70 \%$ similarity over 10 aa to replication protein E1 of papilloma virus strains 28 and 18. It also had $45 \%$ similarity and $73 \%$ similarity over 11 aa to the E2L polyprotein of vaccinia and variola (HHV6) viruses. No sequence similarities were detected with the remaining three epitope peptides, aa 713-728, 745-760, and 889-904.

\section{Discussion}

Thirteen peptides within the intracytoplasmic domain of IA-2, all of which could be presented by HLA-DR4 encoded by the DR4-DQ8 haplotype, elicited T-cell responses in at-risk relatives. The overlap of these peptides suggests that the number of epitopes was possibly nine. Five peptides, between aa 799-934, elicited responses in relatives bearing either the DR4-DQ8- or DR3DQ2-susceptible HLA haplotypes. The remaining peptides elicited responses only in relatives bearing the DR4-DQ8 haplotype. At least two sources of these epitopes are indicated by the very high degree of homology between the two tyrosine phosphatases, IA-2 and IAR.

Interestingly, four peptides elicited responses in the DR3-DQ2 homozygous control, and one of these four also elicited responses in the DR4DQ8 homozygous control. Other evidence demonstrates that $\mathrm{T}$ cells in normal individuals are capable of reacting to autoantigens $(9,15-17)$. The important inference, however, is that these four epitope peptides (shared sequences VIVM LTPLVE, EDFLVRSFYL) should contain the strongest clues to cross-reactive epitopes, e.g., in environmental agents that could trigger or exacerbate islet autoimmunity.

The contribution of environment to type 1 diabetes can be gauged from the lack of concordance for disease in the majority of identical twins (18). However, the environmental factors responsible remain enigmatic. Some viruses such as Coxsackievirus (19) and rubella (20), as well as the rodenticide "Vacor" (21), directly damage pancreatic islet $\beta$-cells and are associated with $\beta$-cell autoimmunity, but such examples are rare, and evidence for persisting infection of $\beta$-cells is lacking (22). Infectious agents could also trigger $\beta$-cell autoimmunity indirectly (re- 
viewed in ref. 23), particularly by activating $\mathrm{T}$ cells cross-reactive with islet proteins, a mechanism termed molecular mimicry.

Molecular mimicry has been proposed between the islet autoantigen glutamic acid decarboxylase 65 (GAD65) (aa 257-273) and the P2C protein of Coxsackievirus B4, which share $59 \%$ identity and $76 \%$ similarity over 17 aa (24). This peptide from GAD65 elicits T-cell responses in humans with type 1 diabetes (17) and in the nonobese diabetic (NOD) mouse model (25). Tcell responses to Coxsackie virus B (strain unstated) have been reported in recently diagnosed type 1 diabetes (14). However, the only reasonably direct evidence for molecular mimicry in type 1 diabetes is the experimental demonstration that infection with lymphocytic choriomeningitis virus (LCMV) triggers immune-mediated $\beta$-cell destruction in mice that transgenically express an LCMV glycoprotein or nucleoprotein in their $\beta$-cells $(26,27)$.

Evidence for a role of viral infection close to diagnosis of type 1 diabetes is the finding that IgM responses to Coxsackievirus (28) and T-cell responses to both Coxsackievirus and adenovirus, but not to the herpesviruses, or to mumps, polio, tick-borne encephalitis virus, or rotavirus (14), were higher in people at diagnosis than in controls. The dominant IA-2 epitope peptide aa 805-820 has high identity and similarity over $8-11$ aa to sequences within several viruses. The nonamer in this peptide predicted to bind to DR4 (11) is in fact VIVMLTPLV. The most likely anchor residues for binding [Table $1(10,11)]$ are unbolded; the bolded residues are therefore most likely to be T-cell receptor contact residues (TCRCR) potentially critical for molecular mimicry. The strongest similarity is with the VP7 protein of human rotavirus (serotype 3 , strain $P$, reovirus family). VP7 contains the sequence IIVILSPLL (aa 41-49) with identical TCR-CR; although the anchor residues differ, they are equally effective for DR4 binding (11). By using HLA-DQ8 $\left({ }^{*} 0302\right)$ binding peptides curated in the MHCPEP Database (29) to derive a matrix for DQ8 (Honeyman, Brusic, Harrison, manuscript in preparation), two overlapping decamers in the same IA-2 region were also predicted to bind to DQ8 (Table 1), which is consistent with the high T-cell responses seen to this epitope. The first DQ8 frame, IIVILSPLLN, has $100 \%$ similarity to VP7 in its potential TCR-CR. VP7 is one of the two immunogenic proteins that confer serotype specificity and is currently being used by others to develop a rotavirus vaccine.
Jones and Crosby (14) noted a sequence similarity between GAD 65 (aa 108-137) and rotaviral VP7 protein, although they could not elicit increased $\mathrm{T}$-cell responses to whole rotavirus (strain unstated) in people with recently diagnosed type 1 diabetes. The cited GAD65 sequence contains a T-cell epitope peptide, MNILLQYVVKSFDRST (aa 115-130, with 88\% homology to GAD67, aa 121-136), in mice transgenic for human HLA-DR4 (30). We have identified this epitope (aa 115-129) in at-risk relatk; 4tives and healthy controls homozygous for DR4-DQ8 (Honeyman, Stone and Harrison, unpublished data). The predicted DR4-binding nonamer within the GAD65 peptide is ILLQYV VKS, and for VP7 it is VLLNYVLKS; in GAD 67 the equivalent region is ILLNYVRKT. GAD65 therefore has $100 \%$ similarity and $80 \%$ identity with VP7 in the potential TCR-CR. The region of VP7 containing both sequence similarities is immunologically interesting. It contains many hydrophobic potential anchor residues for HLA class II molecules and an epitope for cytotoxic $\mathrm{T}$ cells in C57/B 16 mice immunized with rotavirus (31), adjacent to the sequences with similarity to GAD65 and IA-2. The GAD and IA-2 similarities raise the interesting possibility that rotavirus infection could simultaneously activate $T$ cells to two type 1 diabetes autoantigens (see also below).

Rotavirus is a major enteric pathogen of early childhood that causes regular winter outbreaks of gastroenteritis in daycare centers. Children can have multiple infections by different serotypes. Early-age daycare was found to confer increased risk for type 1 diabetes (32), consistent with a link between rotavirus and type 1 diabetes. Serologically, herd immunity is almost complete by age 5 (33). Nevertheless, proliferative CD4 T-cell responses have been detected in humans within 4 to 6 weeks following rotavirus reinfection (34). These CD4 $\mathrm{T}$ cells were of the CD45RA-negative (memory), $\alpha 4 \beta 7$ integrinhigh subset, indicating that gastrointestinal immune responses generate $\alpha 4 \beta 7$-positive T-cell memory. An interesting convergence is that GAD-responsive $T$ cells from people with recently diagnosed type 1 diabetes are $\alpha 4 \beta 7$ positive (35) and T cells in the early phase of insulitis in NOD mice are $\beta 7$-integrin high (36). These data suggest that rotavirus-responsive CD4, $\beta 7$ positive $\mathrm{T}$ cells could migrate selectively to the islets. The similarities of the other viruses with peptide VIVMLTPLVEDGVKQC and with the other IA-2 epitope peptides include anchor resi- 
dues for DR4(*0401), but the potential TCR-CR are not quite as remarkable as for rotavirus.

IA-2 epitope peptide aa 919-934, as well as being similar to the surface glycoprotein of herpes simplex virus, has $60 \%$ identity and $80 \%$ similarity over 10 aa that include the predicted DR4-binding nonamer ILIDMVLNR, with bovine kappa casein YIPIQYVLSR (aa 26-35), although the similarity of the potential TCR-CR is only $40 \%$. While the role of bovine milk proteins as potential aetiologic agents in type 1 diabetes is controversial (37), T-cell responses to whole casein have been reported in type 1 diabetes (38). There is also a high similarity of the common sequence EDFLVRSFYL (aa 847-856) of the IA-2 epitope peptides encompassing aa 841-898 with sequences in wheat and broad bean proteins. Peptide aa 841-856 contains a DR4-binding motif WCEDFLVRS (cf. VLNDFLVRS in wheat and beans) and a predicted DQ8 binding motif IWCEDFLVRS (cf. RVLNDFLVRS in wheat and beans). The class II MHC molecule of NOD mice, $\mathrm{I}-\mathrm{A}^{\mathrm{g7}}$, is the structural counterpart of human DQ8(*0302), and NOD mice fed casein supplement (L. C. Harrison, unpublished results), wheat flour, and to a lesser extent, soya bean meal (39), have an accelerated onset of diabetes.

In conclusion, we have identified T-cell epitope peptides in the intracytoplasmic domain of the type 1 diabetes autoantigen, tyrosine phosphatase IA-2, whose sequence analysis suggests that immunity to rotavirus (whose VP7 sequence mimics epitopes in both IA-2 and GAD) and possibly other viruses and dietary proteins could predispose to type 1 diabetes by activating cross-reactive $T$ cells. Further studies are now required to directly test this hypothesis.

\section{Acknowledgments}

We are grateful to the volunteers for their generous blood donations, and to Dr. Barbara Coulson and Mr. Vladimir Brusic for helpful discussions, Dr. Brian Tait for tissue typing, Dr. Robert Schmidli and Dr. Peter Colman for autoantibody assays, and Mrs. Margaret Thompson for secretarial assistance. N.L.S. is supported by Vic Health, M.C.H. and L.C.H. by the National Health \& Medical Research Council of Australia and The Angelo and Susan Alberti Program Project Grant from the Juvenile Diabetes Foundation International.

\section{References}

1. Rabin DU, Pleasic SM, Shapiro JA, et al. (1994) Islet cell antigen 512 is a diabetes-specific islet autoantigen related to tyrosine phosphatases. J. Immunol. 152: 3183-3188.

2. Lan MS, Lu J, Goto Y, Notkins AL. (1994) Molecular cloning and identification of a receptor-type protein tyrosine phosphatase, IA-2, from human insulinoma. DNA Cell Biol. 13: 505-514.

3. Solimena M, Dirkx R, Hermel JM, et al. (1996) ICA512, an autoantigen of type I diabetes, is an intrinsic membrane protein of neurosecretory granules. EMBO J. 15: 2102-2114.

4. Bonifacio E, Lampasona V, Genovese S, Ferrari M, Bosi E. (1995) Identification of protein tyrosine phosphatase-like IA-2 (islet cell antigen 512) as the insulin-dependent diabetes-related $37 / 40 \mathrm{~K}$ autoantigen and a target of islet-cell antibodies. J. Immunol. 155: 5419-5426.

5. Cui L, Wei-Ping Y, deAizpurua HJ, Schmidli RS, Pallen CJ. (1996) Cloning and characterization of islet cell antigen-related protein-tyrosine phosphatase (PTP), a novel receptor-like PTP and autoantigen in insulin-dependent diabetes. J. Biol. Chem. 271: 24817-24823.

6. Notkins AL, Lu J, Qing L, et al. (1996) IA-2 and IA-2 $\beta$ are major autoantigens in type 1 diabetes and the precursors of the $40 \mathrm{kDa}$ and $37 \mathrm{kDa}$ tryptic fragments. J. Autoimmun. 9: 677-672.

7. Hawkes CJ, Wasmeier C, Christie MR, Hutton JC. (1996) Identification of the $37-\mathrm{kDa}$ antigen in type 1 diabetes as a tyrosine phosphatase-like protein (phogrin) related to IA-2. Diabetes 45: 11871192.

8. Schmidli RS, Colman PG, Cui L, et al. (1998) Antibodies to the novel protein tyrosine phosphatase IAR predict development of insulin-dependent diabetes mellitus (IDDM) in first-degree relatives at-risk for IDDM. Autoimmunity (in press).

9. Durinovic-Bello I, Hummel M, Ziegler AG. (1996) Cellular immune responses to diverse islet cell antigens in type 1 diabetes. Diabetes 45: 795-800.

10. Hammer J, Bono E, Gallazzi F, Belunis C, Nagy Z, Sinigaglia F. (1994) Precise prediction of MHC class II-peptide interaction based on peptide sidechain scanning. J. Exp. Med. 180: 2353-2358.

11. Brusic V, Rudy G, Honeyman MC, Hammer J, Harrison LC. (1998) Prediction of MHC class IIbinding peptides using an evolutionary algorithm and artificial neural network. Bioinformatics 14: 1-9.

12. Sinigaglia $F$, Romagnoli $P$, Guttinger $M$, Takacs $B$, Pink JRL. (1991) Selection of T-cell epitopes and vaccine engineering. Methods Enzymol. 203: 370386.

13. Harrison LC, Honeyman MC, Trembleau S, et al. (1997) A peptide-binding motif for I-Ag7, the class II major histocompatibility complex (MHC) 
molecule of NOD and Biozzi $\mathrm{AB} / \mathrm{H}$ mice. J. Exp. Med. 185: 1013-1023.

14. Jones DB, Crosby I. (1996) Proliferative lymphocyte responses to virus antigens homologous to GAD65 in type 1 diabetes. Diabetologia 39: 13181324.

15. Rudy G, Stone N, Harrison L, et al. (1995) Similar peptides from two $\beta$-cell autoantigens, proinsulin and GAD, stimulate T cells of individuals at risk for IDDM. Mol. Med. 1: 625-633.

16. Van Eden W, Anderton SM, Van der Zee R, Prakken BJ, Broeren CP, Wauben MH. (1996) (Altered) self peptides and the regulation of selfreactivity in the peripheral T-cell pool. Immunol. Rev. 149: 55-73.

17. Schloot NC, Roep BO, Wegmann DR, Yu L, Wang TB, Eisenbarth GS. (1997) T-cell reactivity to GAD65 peptide sequences shared with Coxsackie virus protein in recent-onset type 1 diabetes, postonset type 1 diabetes patients and control subjects. Diabetologia 40: 332-338.

18. Kumar D, Gemayel NS, Deapen D, et al. (1993) North-American twins with type 1 diabetes. Genetic, etiological, and clinical significance of disease concordance according to age, zygosity, and the interval after diagnosis of the first twin. Diabetes 42: 1351-1363.

19. Yoon JW, Austin M, Onodera T, Notkins AL. (1979) Virus-induced diabetes mellitus. Isolation of a virus from the pancreas of a child with diabetic ketoacidosis. N. Engl. J. Med. 300: 1173-1179.

20. Forrest JM, Menser MA, Burgess JA. (1971) High frequency of diabetes mellitus in young adults with congenital rubella. Lancet 2 (720): 332-334.

21. Karam JH, Lewitt PA, Young CW. (1980) Insulinopenic diabetes after rodenticide (Vacor) ingestion. A unique model of acquired diabetes in man. Diabetes 29: 971-978.

22. Foulis AK, McGill M, Farquharson MA, Hilton DA. (1997) A search for evidence of viral infection in pancreases of newly diagnosed patients with type 1 diabetes. Diabetologia 40: 53-61.

23. Harrison LC, McColl G. (1998) Infection and autoimmune disease. In: The Autoimmune Diseases, 2nd ed. Rose NR, Mackay IR (eds). Academic Press, San Diego, pp. 127-140.

24. Atkinson MA, Bowman MA, Campbell L, Darrow BL, Kaufman DL, Maclaren NK. (1994) Cellular immunity to a determinant common to glutamate decarboxylase and coxsackie virus in insulin-dependent diabetes mellitus. J. Clin. Invest. 94: 21252129.

25. Tian J, Lehmann PV, Kaufman DL. (1994) T cell cross-reactivity between coxsackievirus and glutamic acid decarboxylase is associated with a murine diabetes susceptibility allele. J. Exp. Med. 180: 1979-1984.

26. Ohashi P, Oehen S, Buerki K, et al. (1991) Ablation of "tolerance" and induction of diabetes by virus infection in viral antigen transgenic mice. Cell 65: 305-317.

27. Oldstone MBA, Nerenberg $M$, Southern $P$, Price $J$, Lewicki H. (1991) Virus infection triggers insulindependent diabetes mellitus in a transgenic model: Role of anti-self (virus) immune response. Cell 65: 319-331.

28. Hyoty H, Hiltunen M, Knip M, et al. Akerblom HK and Childhood Diabetes in Finland (DiMe) Study Group. (1995) A prospective study of the role of Coxsackie $B$ and other enterovirus infections in the pathogenesis of type 1 diabetes. Diabetes 44: 652-657.

29. Brusic V, Rudy G, Kyne AP, Harrison LC. (1996) MHCPEP-a database of MHC-binding peptides: Update 1995. Nucl. Acids Res. 24: 242-244.

30. Patel SD, Cope AP, Congia M, et al. (1997) Identification of immunodominant $\mathrm{T}$ cell epitopes of human glutamic acid decarboxylase 65 by using HLA-DR $(A 1 * 0101, \mathrm{Bl} * 0401)$ transgenic mice. Proc. Natl. Acad. Sci. U.S.A. 94: 8082-8087.

31. Heath R, Stagg S, Xu F, McCrae MA. (1997) Mapping of the target antigens of the rotavirus-specific cytotoxic T-cell response. J. Gen. Virol. 78: 10651075.

32. Verge CF, Howard NJ, Irwig L, Simpson JM, Mackerras D, Silink M. (1994) Environmental factors in childhood IDDM. A population-based, case-control study. Diabetes Care 17: 1381-1389.

33. Bishop RF, Unicomb LE, Barnes GL. (1991) Epidemiology of rotavirus serotypes in Melbourne, Australia, from 1973 to 1989. J. Clin. Microbiol. 29: 862-868.

34. Rott LS, Rose JR, Bass D, Williams MB, Greenberg HB, Butcher EC. (1997) Expression of mucosal homing receptor $\alpha 4 \beta 7$ by circulating $\mathrm{CD}^{+}$cells with memory for intestinal rotavirus. J. Clin. Invest. 100: 1204-1208.

35. Paronen J, Klemetti P, Kantele JM, et al. (1997) Glutamate decarboxylase-reactive peripheral blood lymphocytes from patients with IDDM express gut-specific homing receptor $\alpha 4 \beta 7$-integrin. Diabetes 46: 583-588.

36. Hanninen A, Salmi M, Simell O, Jalkanen S. (1996) Mucosa-associated (beta 7-integrin high) lymphocytes accumulate early in the pancreas of NOD mice and show aberrant recirculation behaviour. Diabetes 45: 1173-1 180.

37. Harrison LC. (1996) Cows' milk and type 1 diabetes [Commentary]. Lancet 348: 905-906.

38. Cavallo MG, Fava D, Monetini L, Barone F, Pozzilli P. (1996) Cell-mediated immune response to beta casein in recent-onset insulin-dependent diabetes: Implications for disease pathogenesis. Lancet 348: 926-928.

39. Hoorfar J, Buschard K, Dagnaes-Hansen F. (1993) Prophylactic nutritional modification of the incidence of diabetes in autoimmune nonobese diabetic (NOD) mice. Br. J. Nutrition 69: 597-607. 